\title{
Área do limbo foliar da castanheira-do-Brasil por medidas lineares
}

\author{
Luana BOUVIÉ ${ }^{*}$, Andréa Carvalho da SILVA², Daniela Roberta BORELLA ${ }^{1}$, \\ Cátia Cardoso da SILVA³, Mariana PIZZATTO2
}

\author{
1 Programa de Pós-Graduação em Física Ambiental, Universidade Federal de Mato Grosso, Cuiabá, MT, Brasil. \\ (ORCID: *; 0000-0003-2941-2116) \\ 2Instituto de Ciências Agrárias e Ambientais, Universidade Federal de Mato Grosso, Sinop, MT, Brasil. \\ (ORCID: 0000-0003-2921-3379; 0000-0001-5751-0368) \\ 3 Programa de Pós-Graduação em Ciência Florestal, Universidade Federal de Viçosa, Viçosa, MG, Brasil. (ORCID: 0000-0002-1079-8728) \\ E-mail: bouvieluana@gmail.com (ORCID: 0000-0003-1884-9347)
}

Recebido em 27/06/2019; Aceito em 24/04/2020; Publicado em 20/05/2020.

\begin{abstract}
RESUMO: Neste trabalho foram gerados e validados modelos de estimativa da área da folha da espécie Bertholletia excelsa Bonpl. (castanheira-do-Brasil) a partir das medidas lineares do limbo foliar. Foram coletadas 1500 folhas em diferentes posições da copa de árvores adultas e jovens (em função do ciclo reprodutivo), sendo usadas 1000 e 500 folhas para calibração e validação estatísticas, respectivamente. Foram obtidos como medidas do limbo da folha: comprimento (C), largura (no centro da folha, na base e no ápice) e a área foliar real (AFR). A avaliação do desempenho estatístico (validação) foi realizada pelos indicativos erro médio (MBE), raiz quadrática do erro médio (RMSE) e índice de ajustamento de Willmott (dW). Apenas a medida de largura no centro da folha e do comprimento são suficientes para estimar a área da folha de B. excelsa., que pode ser dada pela equação $\mathrm{AF}=\left\{0,8743^{*}\left\{\left(\mathrm{C}^{*} \mathrm{~L}\right)^{0,9790}\right]\right\}-1,84$, independentemente da posição da folha e da idade planta.

Palavras-chave: Bertholletia excelsa; análise de regressão; indicativos estatísticos.
\end{abstract}

\section{Field of the limbo foliar of castanheira-do-Brasil with linear measures}

\begin{abstract}
In this work, models of estimation of the leaf area of the species Bertholletia excelsa Bonpl. (Brazil nut) from the linear measurements of the leaf blade. 1500 leaves were collected in different positions of the crown of adult and young trees (depending on the reproductive cycle), using 1000 and 500 leaves for calibration and statistical validation, respectively. Leaf length (C), width (at the center of the leaf, at the base and at the apex) and leaf area (AFR) were obtained as measures of leaf limb. Statistical performance evaluation (validation) was performed using the mean error (MBE), root mean square error (RMSE) and Willmott's index of adjustment $(\mathrm{dW})$. Only the width measure at the center of the leaf and the length are sufficient to estimate the area of the leaf of $B$. excelsa., Which can be given by the equation $\mathrm{AF}=\{0.8743 *\{(\mathrm{C} * \mathrm{~L}) 0.9790]\}-1.84$, regardless of leaf position and plant age.
\end{abstract}

Keywords: Bertholletia excelsa; regression analysis; statistical indicatives.

\section{INTRODUÇÃO}

B. excelsa Bonpl. destaca-se ecológica, econômica e socialmente, como símbolo da produção florestal não madeireira e do desenvolvimento sustentável e estratégico para a conservação da floresta amazônica. Ocorre naturalmente em matas de terra firmes da bacia amazônica e das Guianas (em agrupamentos denominados de castanhais); é uma árvore dominante que influência na dinâmica das clareiras e na sucessão ecológica na Amazônia (DUCHELLE et al., 2011).

A espécie apresenta altas taxas de crescimento inicial em sistemas agroflorestais ou em plantios homogêneos para recuperação e/ou reposição florestal (FERREIRA et al., 2012). Nesse contexto, é crescente a demanda de informações acerca da sua fenologia e do desenvolvimento, para subsidiar tanto o cultivo/coleta, quanto o manejo da sua diversidade genética (NEVES et al., 2016).

$\mathrm{Na}$ região amazônica, existe grande interesse no recrutamento e na regeneração de plântulas de castanheirado-Brasil, mediante a importância da colheita ao longo prazo e da sustentabilidade. Informações acerca da sua perpetuação natural ainda são necessárias, visto que, o desenvolvimento e a maturação dos frutos, bem como a regeneração dos castanhais, são lentos e altamente dependentes de características bióticas e abióticas específicas (SCOLES et al., 2016).

A quantificação do crescimento e o desenvolvimento da planta em escala temporal, pode ser considerado como um dos primeiros passos para entender o comportamento de qualquer espécie, pois explica como o ambiente influencia no incremento de massa seca e como condiciona a partição dos fotoassimilados, dentre outras respostas fisiológicas das plantas.

A área foliar pode ser considerada como um dos mais importantes parâmetros quantitativos em análise de crescimento de plantas, pois a assimilação do carbono está relacionada linearmente com a área de interceptação dos fótons, e além disso, a folha é o principal órgão responsável pelas trocas gasosas entre a planta e o ambiente. Logo, a área foliar possibilita a estimativa da perda de água por 
transpiração (FAVARIN et al., 2002), e pode ser empregada na definição e avaliação de técnicas culturais como poda, adubação, espaçamentos e na densidade de plantio (OLIVEIRA et al., 2015), avaliação dos danos causados por doenças e pragas foliares (FAVARIN et al., 2002), determinação do volume da calda de defensivos agrícolas (FERREIRA et al., 2010), dentre outros.

$\mathrm{Na}$ determinação da área foliar podem ser empregados métodos diretos ou indiretos. Os métodos diretos são destrutivos e requerem uso de equipamentos com custos elevados, enquanto que os métodos indiretos, são considerados como não destrutivos, apresentam facilidades de uso, baixo custo e podem fornecer estimativas precisas durante o ciclo da planta, pois reduzem a variabilidade associada a procedimentos de amostragem.

Em geral, segundo Benincasa (2003), os métodos nãodestrutivos permitem avaliações sucessivas do crescimento e da expansão foliar da mesma planta até o final do ciclo, além de ser rápido e de menor custo. A quantificação nãodestrutiva da área foliar de forma mais simplificada, se dá por meio de equações estatísticas ajustadas com base em medidas lineares, desenvolvidas para cada espécie ou características do limbo foliar, calibradas e validadas estatisticamente com base na superfície foliar real (SCHMILDT et al., 2016).

Nos últimos anos, foram desenvolvidos vários modelos para espécies arbóreas como laranjeira (MAZZINI et al., 2010), mangueira (LIMA et al., 2012), figueira (SOUZA et al., 2014) e pessegueiro (SACHET et al., 2015), coníferas (JONES et al., 2015), teca (LEROY et al., 2007; TONDJO et al., 2015; SILVA et al., 2020), braúna e craibeira (QUEIROZ et al., 2013), para dezenove espécies lenhosas subtropicais (MENG et al., 2015). Para espécies florestais tropicais, podem ser destacados os trabalhos de Cabanez et al. (2014), Monteiro et al., (2014) e Monteiro et al., (2017).

O formato geométrico do limbo foliar é uma característica morfológica específica de cada espécie vegetal, e a relação entre as dimensões lineares e a área foliar, depende da quantidade de recortes na borda do limbo foliar (PINTO et al. 2008), indicando que, é necessário a geração de modelos específicos de estimativa da área foliar para cada espécie vegetal, e que dentro da mesma espécie, podem ser gerados diferentes modelos de estimativa de acordo com a diferenciação morfológica existente entre diferentes idades da planta (LIU et al., 2017; SOUZA et al., 2014). Martin; Thomas (2013) encontraram diferenças significativas entre folhas de mudas e árvores adultas de Dacryodes excelsa e Miconia mirabilis. Brito-Rocha et al. (2016), também obtiveram diferenças em estimativas da área do limbo de folhas nos estádios ontogenéticos de Psidium cattleyanum e Tabebuia stenocalyx com estrutura e área foliar.

Especificamente, estimativas da área foliar durante o desenvolvimento da castanheira-do-Brasil são úteis para diversos estudos agronômicos, ecológicos e biológicos. Entretanto, análises no interior de florestas maduras ou em áreas de regeneração, tornam-se inviáveis por métodos destrutivos, e quando, associados aos baixos índices de velocidade e percentual de germinação da espécie, requerem mesmo em viveiro, que sejam empregados métodos indiretos (CUSI-AUCA et al., 2018). Diante disso, este trabalho objetivou gerar e validar estatisticamente, modelos de estimativa da área do limbo da folha de Bertholletia excelsa Bonpl., por meio de medidas lineares de folhas.

\section{MATERIAL E MÉTODOS}

As coletas das folhas de matrizes de B. excela ocorreram em agosto de 2016, na região de Sinop-MT, norte do Estado do Mato Grosso (Transição Cerrado-Amazônia). Pela classificação de Köppen, a região apresenta clima Aw, tipo tropical quente e úmido, possuindo duas estações bem definidas: chuvosa (entre os meses de outubro e abril) e seca (de maio a setembro); com baixa amplitude térmica anual (médias mensais entre 24 e $27^{\circ} \mathrm{C}$ ) e precipitação média anual em torno de $1970 \mathrm{~mm}$ (SOUZA et al., 2013).

Foram selecionadas seis matrizes da espécie $B$. excelsa que possuíam bom estado fitossanitário, classificadas em função da presença/ausência dos resquícios das estruturas reprodutivas abaixo das copas, em "Árvores Jovens" - AJ (que não haviam iniciado ciclos reprodutivos) e "Árvores Adultas" - AA (que completaram ciclos reprodutivos com dispersão de frutos no ciclo 15/16) (Figura 1; Tabela 1).

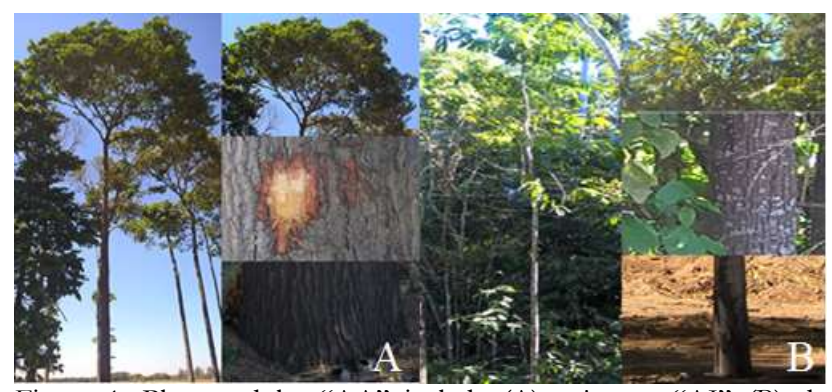

Figura 1. Planta adulta "AA" isolada (A) e jovem "AJ" (B) de castanheira-do-Brasil com sua copa, ritidoma e base, na região de Sinop-MT.

Figure 1. Adult plant "AA" (A) isolated and young "AJ" (B) of Brazil nut tree with its crown, ritidoma and base, in the region of Sinop-MT.

Tabela 1. Diâmetro a altura do peito (DAP); altura até a inserção do primeiro galho $(\mathrm{HC})$ e altura total $(\mathrm{HT})$ das matrizes de B. excelsa, consideradas para coletas das folhas.

Table 1. Diameter at breast height (DAP); height up to the insertion of the first branch (HC) and total height (HT) of the B. excelsa matrices, considered for leaf collection.

\begin{tabular}{ccccc}
\hline & Matriz & DAP $(\mathrm{cm})$ & HC $(\mathrm{m})$ & HT $(\mathrm{m})$ \\
\hline \multirow{4}{*}{ AJ } & 1 & 30,88 & - & 10,9 \\
& 2 & 18,08 & - & 8,8 \\
& 3 & 9,87 & - & 8,8 \\
\hline \multirow{3}{*}{ AA } & 1 & 27,06 & 7,9 & 28,6 \\
& 2 & 35,33 & 7,9 & 19,1 \\
& 3 & 27,69 & 6,9 & 14,9 \\
\hline
\end{tabular}

Foram coletadas 250 folhas completamente expandidas por matriz (perfazendo 1500 folhas), não danificadas, sem deformações e de diferentes tamanhos, para permitir maior variabilidade quanto às dimensões e formas das folhas em pleno desenvolvimento vegetativo. As folhas foram coletadas de forma homogênea na copa de três árvores diferentes dentro de cada classe de idade.

Com auxílio de réguas milimetradas e fitas métricas foram obtidas as medidas lineares (em cm) das folhas (o pecíolo das folhas foi desconsiderado): comprimento $(\mathrm{C})$, largura central, da base e do ápice (LC, LB e LA, respectivamente); LB e LA foram mensuradas dois centímetros antes das extremidades do limbo foliar (Figura 2). Após as aferições dos parâmetros lineares, determinou-se a área foliar real (AFR), em $\mathrm{cm}^{2}$, com

Nativa, Sinop, v. 8, n. 3, p. 381-389, mai./jun. 2020. 
medidor fotoelétrico Licor, Modelo LI-3000. O equipamento foi calibrado a cada 200 análises, tendo como referência a área de folhas de papel A4.

Os conjuntos das folhas para as duas idades de matrizes (AA e AJ), foram separados em três classes com base na distribuição de frequência da AFR, para minimizar o desvio padrão das medidas lineares e da área do limbo foliar.

C

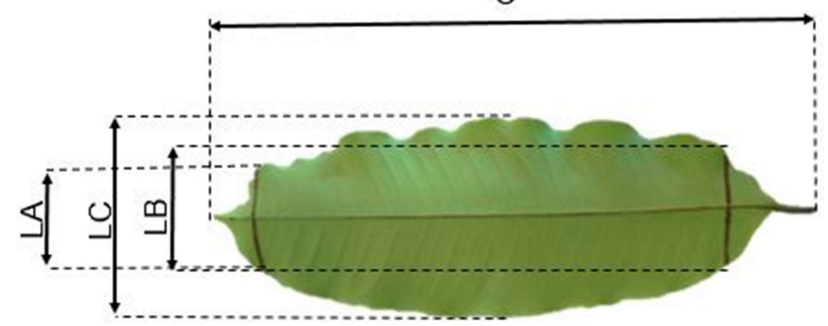

Figura 2. Representação das medidas lineares do limbo de folhas de B. excelsa. (C - comprimento; LA - largura do ápice; LC - largura no centro e LB - largura da base).

Figure 2. Representation of linear measures of leaf blade of $\mathrm{B}$. excelsa (C - length; LA - apex width; LC - width at the center and LB - width of the leaf base).

Com base nas medidas C, LA, LB e LC foram avaliados 12 modelos estatísticos que descrevem comportamentos linear e polinomial (Tabela 2), adaptados de Souza et al., (2014). Nesse caso, considerou-se a AF como variável dependente e as dimensões lineares (e suas respectivas interações) como variáveis independentes.

Realizou-se o teste F entre os valores de AFR e AFE observados para verificar a homogeneidade dos dados, em conjunto com o teste de normalidade com as variáveis independentes e dependentes (AFR) isoladas, para o conjunto total de dados e para as duas idades das plantas.

Empregou-se a ferramenta de otimização Solver do Microsoft Excel, com objetivo de maximização do coeficiente de determinação $\left(\mathrm{R}^{2}\right)$. Para a geração e avaliação do desempenho estatístico dos modelos foram utilizadas 500 e 250 folhas, para cada idade de planta, respectivamente.

Tabela 2. Modelos analíticos ajustados para a estimativa da área de folhas de árvores jovens e adultas de Bertholletia excelsa a partir de dimensões lineares.

Table 2. Analytical models generated and validated for the estimation of leaf area of young and adult trees of Bertholletia excelsa from linear dimensions.

$\begin{array}{rlrl}1 & \mathrm{AF}=\mathrm{a}_{1} * \mathrm{C} & 7 & \mathrm{AF}=\mathrm{a}_{1} * \mathrm{C} * \mathrm{~L} \\ 2 & \mathrm{AF}=\left(\mathrm{a}_{1} * \mathrm{C}\right)+\mathrm{a}_{2} & 8 & \mathrm{AF}=\left(\mathrm{a}_{1} * \mathrm{C} * \mathrm{~L}\right)+\mathrm{a}_{2} \\ 3 & \mathrm{AF}=\mathrm{a}_{1} * \mathrm{C}^{\mathrm{b}} & 9 & \mathrm{AF}=\mathrm{a}_{1} *(\mathrm{C}+\mathrm{L}) \\ 4 & \mathrm{AF}=\mathrm{a}_{1} * \mathrm{~L} & 10 & \mathrm{AF}=\left[\mathrm{a}_{1} *(\mathrm{C}+\mathrm{L})\right]+\mathrm{a}_{2} \\ \mathbf{5} & \mathrm{AF}=\left(\mathrm{a}_{1} * \mathrm{~L}\right)+\mathrm{a}_{2} & 11 & \mathrm{AF}=\mathrm{a}_{1} *\left[(\mathrm{C} * \mathrm{~L})^{\mathrm{b}}\right] \\ \mathbf{6} & \mathrm{AF}=\mathrm{a}_{1} *\left(\mathrm{~L}^{\mathrm{b}}\right) & 12 & \mathrm{AF}=\mathrm{a}_{1} *\left[(\mathrm{C}+\mathrm{L})^{\mathrm{b}}\right]\end{array}$

Considerou-se $\mathrm{AF}$ é a área foliar estimada pela fórmula $\left(\mathrm{cm}^{2}\right) ; \mathrm{C}$ o comprimento da folha $(\mathrm{cm})$; L a largura da folha $(\mathrm{cm})$ e a1, a2 e b são coeficientes ajustados.

As equações que consideraram a largura da folha (números 4 a 12 da Tabela 2) foram ajustadas considerando apenas LC e LM (largura média, obtida pela média aritmética simples de LC, LB e LA). Posteriormente, foram estabelecidas regressões lineares entre AFE com LC (variável dependente), e ainda AFE com LM (variável dependente) para análise do erro padrão da estimativa, coeficiente angular e o coeficiente de determinação $\left(\mathrm{R}^{2}\right)$, visando avaliar a necessidade de inclusão das três larguras mensuradas.

Para avaliar o desempenho dos modelos calibrados, foram calculados os indicativos estatísticos: $\mathrm{MBE}$ (mean bias error-erro médio relativo, equação 1); RMSE (root mean square error - raiz quadrada do quadrado médio do erro, equação 2); $d w$ (índice de ajustamento de Willmott, equação 3) (SOUZA et al., 2014; MONTEIRO et al., 2017).

$$
\begin{array}{ll}
M B E=\frac{\sum_{i=1}^{n}\left|O_{i}-P_{i}\right|}{n} & (\text { Equação 1) } \\
R M S E=\left[\frac{\sum_{i=1}^{n}\left(O_{i}-P_{i}\right)^{2}}{n}\right]^{0,5} & (\text { Equação 2) } \\
d W=1-\frac{\sum_{i=1}^{n}\left(O_{i}-P_{i}\right)^{2}}{\sum_{i=1}^{n}\left(\left|P_{i}-\bar{O}\right|+\left|O_{i}-\bar{O}\right|\right)^{2}} & (\text { Equação 3) }
\end{array}
$$

em que: Pi: valores estimados, em $\mathrm{cm}^{2}$; Oi: valores observados, em $\mathrm{cm}^{2}$; n: número de observações; O: média dos valores observados,

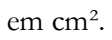

Considerando os indicativos supracitados e no intuito de analisar com maior restrição o desempenho dos modelos gerados, empregou-se o método dos valores ponderados (Vp) dos indicativos estatísticos de acordo com Abreu et al., (2018). Na obtenção do Vp, atribuiu-se pesos de 1 a "n" para cada indicador estatístico em cada modelo, onde " $n$ " corresponde ao número de modelos testados. Ao final, o melhor modelo considerado foi aquele que apresentou $\mathrm{O}$ menor somatório de pesos (menor Vp acumulado).

O método do Vp permite classificar e definir o melhor modelo para a estimativa da área foliar para as espécies estudadas, tendo em vista que considera as informações e particularidades correspondentes a todos os indicativos estatísticos calculados, fornecendo, portanto, um resultado confiável (ABREU et al., 2018). Determinados os modelos mais eficientes (com base no $\mathrm{Vp}$ ), foi realizado novos ajustes, agrupando todas as folhas independentemente da idade da planta e variações morfológicas das folhas de castanheira-doBrasil.

\section{RESULTADOS}

As Figuras 3 e 4 apresentam a descrição estatística por meio de Boxplot e distribuição de frequência relativa (histograma e distribuição normal), das variáveis lineares do limbo foliar e AFR, separadas em função da idade da matriz (AA e AJ). Todas as dimensões lineares avaliadas apresentaram padrão com distribuição normal. AFR apontou um deslocamento inferior na curva de distribuição, indicando a ocorrência de um baixo número de folhas com área de limbo foliar superior à $450 \mathrm{~cm}^{2}$, no entanto, para essa variável (AFR), o coeficiente de assimetria foi 0,59 e 0,50 para AJ e AA, indicando normalidade.

Com base na distribuição de frequência de AFR (Figura 3), os limites das três classes utilizadas para padronização do agrupamento de folhas para geração e validação estatística dos modelos, foram: CI - AFR $\leq 150 \mathrm{~cm}^{2}$; CII $-150<$ AFR $<300 \mathrm{~cm}^{2}$; e CIII: AFR $\geq 300 \mathrm{~cm}^{2}$.

Pelas Tabelas 3 e 4 evidencia-se a homogeneidade e a representatividade amostral dentre os agrupamentos de dados foliares, para geração e validação estatística dos modelos. Nesse caso, dentro de cada classe e para todas as variáveis analisadas, os valores da média aritmética $(\mathrm{M})$ e desvio padrão (DV) foram próximos, (em torno de 1,0 e 1,9 
$\mathrm{cm}$, para M e DV, não ultrapassando $1,9 \mathrm{~cm}$, quando compara-se os dados para geração com os dados de validação, nas diferentes classes de tamanho de folha).

No mesmo sentido, AFR apresentou diferenças médias de $16,4 \mathrm{~cm}^{2}$ e $51,3 \mathrm{~cm}^{2}$ e de desvio padrão entre $1,7 \mathrm{~cm}^{2}$ e $12,9 \mathrm{~cm}^{2}$, nas três classes de tamanho de folhas para AJ, quando compara-se as folhas para geração com as folhas de validação dos modelos; enquanto que para $\mathrm{AA}$, diferenças médias das classes entre $2,0 \mathrm{~cm}^{2}$ e $15,6 \mathrm{~cm}^{2}$ e de desvio padrão entre $0,9 \mathrm{~cm}^{2}$ e $18,8 \mathrm{~cm}^{2}$ (Tabelas 3 e 4).
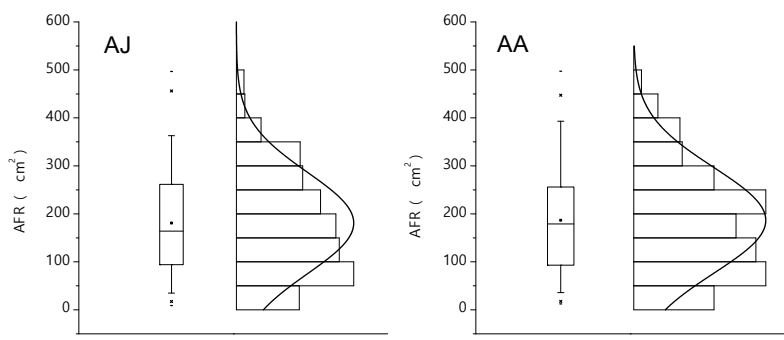

Figura 3. Boxplot e distribuição de frequência relativa da área foliar real $\left(\mathrm{AFR}, \mathrm{em} \mathrm{cm}^{2}\right.$ ) de folhas de matrizes jovens (AJ) e adultas (AA) de Bertholletia excelsa.

Figure 3. Boxplot and relative frequency distribution of the real leaf area $\left(\mathrm{AFR}\right.$, in $\mathrm{cm}^{2}$ ) of leaves of the young matrices (AJ) and adult (AA) of Bertholletia excelsa.
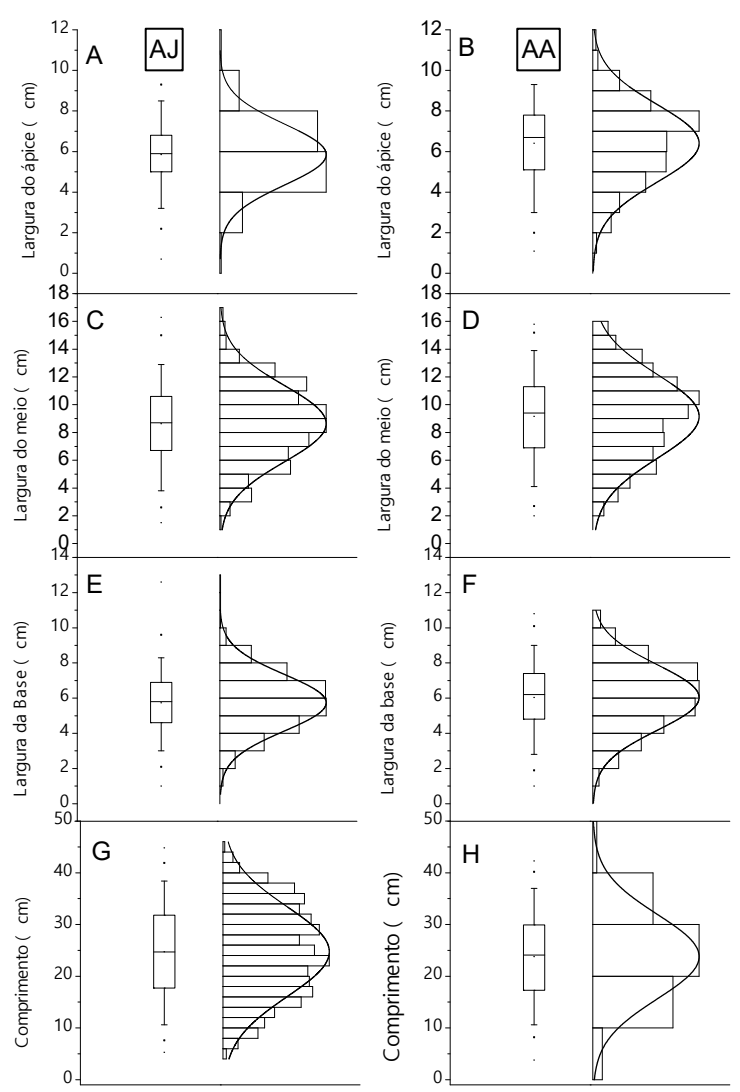

Figura 4. Boxplot e distribuição de frequência relativa representada por histograma e curva normal de comprimento (C), largura do ápice (LA), largura central (LC) e largura da base (LB) de folhas de árvores jovens (AJ) e adultas (AA) de Bertholletia excelsa.

Figure 4. Boxplot and relative frequency distribution represented by histogram and normal curve of the length (C), apex width (LA), width in the center (LM) and width of the base (LB) of leaves of young trees $(\mathrm{AJ})$ and adult trees (AA) of Bertholletia excelsa.
Tabela 3. Variáveis estatísticas de folhas de B. excelsa provenientes de matrizes jovens (AJ), distribuídas em diferentes classes de AFR e agrupadas para geração e validação de modelos estatísticos de estimativa da área do limbo foliar.

Table 3. Statistical variables of leaves of $B$. excelsa from young matrices (AJ), distributed in different classes of AFR and grouped for generation and validation of statistical models of leaf area estimation.

\begin{tabular}{|c|c|c|c|c|c|c|c|c|}
\hline & \multicolumn{4}{|c|}{ Geração } & \multicolumn{4}{|c|}{ Validação } \\
\hline & LI & $\mathrm{M}$ & LS & $\mathrm{DP}$ & LI & $\mathrm{M}$ & LS & $\mathrm{DP}$ \\
\hline \multicolumn{9}{|c|}{ Classe $\mathrm{I}\left(\mathrm{AFR} \leq 150 \mathrm{~cm}^{2}\right)$} \\
\hline $\mathrm{C}$ & 5,3 & 16,1 & 25,3 & 4,3 & 6,4 & 18 & 32,9 & 5,3 \\
\hline LB & 1,0 & 4,3 & 7,1 & 1,2 & 2,6 & 4,8 & 7,2 & 1,0 \\
\hline $\mathrm{LC}$ & 1,5 & 5,9 & 10,0 & 1,8 & 3,0 & 6,6 & 9,5 & 1,5 \\
\hline LA & 0,7 & 4,7 & 8,5 & 1,4 & 2,6 & 5,2 & 8,7 & 1,1 \\
\hline LM & 1,1 & 5,0 & 8,5 & 1,4 & 2,8 & 5,5 & 7,8 & 1,1 \\
\hline AFR & 8,8 & 79,0 & 149,7 & 37,0 & 17,2 & 95,4 & 148,6 & 38,7 \\
\hline \multicolumn{9}{|c|}{ Classe II $\left(150<\right.$ AFR $\left.<300 \mathrm{~cm}^{2}\right)$} \\
\hline $\mathrm{C}$ & 18,1 & 28,3 & 36,8 & 3,9 & 21,1 & 29,2 & 39,2 & 4,4 \\
\hline LB & 4,5 & 6,3 & 8,7 & 0,8 & 3,9 & 6,2 & 8,4 & 1,0 \\
\hline $\mathrm{LC}$ & 7,4 & 9,7 & 13,6 & 1,0 & 6,8 & 10,0 & 12,8 & 1,4 \\
\hline LA & 4,2 & 6,3 & 9,5 & 1,0 & 4,0 & 6,4 & 9,8 & 1,2 \\
\hline LM & 5,7 & 7,4 & 10,1 & 0,8 & 5,5 & 7,5 & 10,3 & 1,0 \\
\hline AFR & 151,0 & 212,8 & 299,1 & 41,0 & 150,4 & 228,2 & 349,5 & 51,7 \\
\hline \multicolumn{9}{|c|}{ Classe III $\left(\right.$ AFR $\left.\geq 300 \mathrm{~cm}^{2}\right)$} \\
\hline $\mathrm{C}$ & 28,5 & 35,8 & 42,8 & 2,9 & 34,5 & 39,2 & 44,8 & 2,5 \\
\hline LB & 6,4 & 7,8 & 9,9 & 0,8 & 5,5 & 8,2 & 12,6 & 1,3 \\
\hline $\mathrm{LC}$ & 9,2 & 12,3 & 16,1 & 1,2 & 10,3 & 12,9 & 16,3 & 1,4 \\
\hline LA & 3,1 & 7,3 & 17,5 & 1,7 & 4,4 & 7,3 & 10,8 & 1,5 \\
\hline LM & 6,6 & 9,1 & 12,7 & 1,0 & 6,7 & 9,5 & 12,5 & 1,3 \\
\hline AFR & 300,1 & 346,4 & 496,7 & 49,0 & 350,1 & 397,7 & 579,1 & 61,9 \\
\hline
\end{tabular}

M: Média; DP: desvio padrão; LS e LI: limite superior e inferior das dimensões lineares $(\mathrm{cm})$ e da área das folhas $\left(\mathrm{cm}^{2}\right)$.

Tabela 1. Variáveis estatísticas de folhas de $B$. excelsa provenientes de matrizes adultas (AA), distribuídas em diferentes classes de AFR e agrupadas para geração e validação de modelos estatísticos de estimativa da área do limbo foliar.

Table 4. Statistical variables of leaves of leaves of $B$. excelsa from adult matrices (AA), distributed in different classes of AFR and grouped for generation and validation of statistical models of estimation of leaf area.

\begin{tabular}{|c|c|c|c|c|c|c|c|c|}
\hline & \multicolumn{4}{|c|}{ Geração } & \multicolumn{4}{|c|}{ Validação } \\
\hline & $\mathrm{LI}$ & $\mathrm{M}$ & LS & DP & LI & $\mathrm{M}$ & LS & $\mathrm{DP}$ \\
\hline \multicolumn{9}{|c|}{ Classe $\mathrm{I}\left(\mathrm{AFR} \leq 150 \mathrm{~cm}^{2}\right)$} \\
\hline $\mathrm{C}$ & 5,1 & 15,8 & 24,6 & 4,2 & 7,2 & 16,7 & 24,9 & 4,3 \\
\hline LB & 1,0 & 4,5 & 7,5 & 1,3 & 1,3 & 4,4 & 7,2 & 1,2 \\
\hline $\mathrm{LC}$ & 2,0 & 6,4 & 10,2 & 1,9 & 2,5 & 6,4 & 9,8 & 1,5 \\
\hline LA & 1,1 & 4,9 & 8,4 & 1,5 & 1,3 & 4,9 & 8,7 & 1,4 \\
\hline LM & 1,6 & 5,3 & 8,6 & 1,5 & 1,7 & 5,2 & 8,0 & 1,3 \\
\hline AFR & 12,3 & 82,7 & 147,6 & 39,0 & 14,9 & 84,7 & 148,9 & 34,2 \\
\hline \multicolumn{9}{|c|}{ Classe II $\left(150<\right.$ AFR $\left.<300 \mathrm{~cm}^{2}\right)$} \\
\hline $\mathrm{C}$ & 3,8 & 26,9 & 36,5 & 3,8 & 19,8 & 27,8 & 33,5 & 3,1 \\
\hline LB & 4,8 & 6,7 & 8,7 & 0,9 & 4,5 & 6,9 & 9,9 & 1,0 \\
\hline $\mathrm{LC}$ & 2,1 & 10,2 & 13,2 & 1,2 & 8,3 & 10,8 & 13,9 & 1,1 \\
\hline LA & 4,4 & 7,3 & 10,3 & 1,1 & 2,9 & 7,3 & 9,7 & 1,3 \\
\hline $\mathrm{LM}$ & 6,0 & 8,1 & 10,4 & 0,8 & 6,1 & 8,3 & 10,5 & 1,0 \\
\hline AFR & 105,5 & 215,6 & 299,6 & 38,8 & 152,0 & 231,2 & 281,2 & 37,9 \\
\hline \multicolumn{9}{|c|}{ Classe III (AFR $\left.\geq 300 \mathrm{~cm}^{2}\right)$} \\
\hline $\mathrm{C}$ & 27,3 & 35,5 & 42,3 & 3,2 & 31,1 & 35,6 & 40,0 & 2,6 \\
\hline LB & 5,7 & 8,3 & 10,4 & 1,0 & 7,5 & 8,9 & 10,8 & 1,0 \\
\hline $\mathrm{LC}$ & 3,5 & 13,1 & 15,8 & 2,0 & 10,4 & 13,4 & 15,5 & 1,0 \\
\hline LA & 5,1 & 8,0 & 17,7 & 1,6 & 6,5 & 9,2 & 11,9 & 1,2 \\
\hline LM & 6,5 & 9,8 & 13,3 & 1,0 & 9,3 & 10,5 & 12,3 & 0,7 \\
\hline AFR & 300,4 & 368,8 & 497,2 & 48,7 & 331,1 & 376,8 & 440,6 & 29,9 \\
\hline
\end{tabular}


As dimensões máximas encontradas para folhas provenientes de AJ foram 44,8; 12,6; 16,3 e 12,5 cm para C, LB, LC e LA, respectivamente, e AFR máxima de $579,1 \mathrm{~cm}^{2}$. Da mesma forma, para AA, observou-se folhas com dimensões lineares máximas de 42,3; 10,8; 15,8; e 17,7 cm para C, LB, LC e LA, respectivamente, e AFR máxima de $497,2 \mathrm{~cm}^{2}$.

$\mathrm{Na}$ Tabela 5 estão expostos os coeficientes angulares (da regressão linear) gerada entre AFE considerando LC (variável independente) e LM (variável dependente). Nesse caso, foram avaliados apenas os modelos que consideram a largura da folha (4 a 12). Apenas os modelos 7 e 11 apresentaram coeficientes angulares menores do que 1,0, permitindo subestimativas de AFE quando se utiliza LM.

Os valores do coeficiente angular e coeficiente de determinação próximos de 1,0, bem como o erro próximo de 0 , indicam que o ajuste das equações utilizando a média de três larguras do limbo foliar (LM) não alterou significativamente AFE (Tabela 5) e não aumentou a precisão na estimativa da área da folha.

Tabela 5. Coeficiente angular (b), erro e coeficiente de determinação $\left(R^{2}\right)$ de regressões lineares entre AFE estimada com LC e com LM, para folhas de matrizes jovens (AJ) e adultas (AA) de B. excelsa.

Table 5. Angular coefficient (b), error and coefficient of determination $\left(\mathrm{R}^{2}\right)$ of linear regressions between estimated EFA with LC and with LM, for leaves of the young (AJ) and adult matrices (AA) of B. excelsa.

\begin{tabular}{ccccccc}
\hline \multicolumn{3}{c}{$\mathrm{AJ}$} & & $\mathrm{AA}$ \\
\hline Equação & $\mathrm{b}$ & Erro & $\mathrm{R}^{2}$ & $\mathrm{~b}$ & Erro & $\mathrm{R}^{2}$ \\
\hline 4 & 1,011 & 0,003 & 0,9405 & 1,020 & 0,003 & 0,9607 \\
5 & 1,013 & 0,003 & 0,9468 & 1,017 & 0,002 & 0,9638 \\
6 & 1,008 & 0,005 & 0,9347 & 1,032 & 0,007 & 0,9430 \\
7 & 0,997 & 0,003 & 0,9862 & 1,006 & 0,002 & 0,9924 \\
8 & 1,000 & 0,002 & 0,9860 & 1,011 & 0,002 & 0,9908 \\
9 & 1,005 & 0,000 & 0,9973 & 1,004 & 0,000 & 0,9990 \\
10 & 1,005 & 0,000 & 0,9973 & 1,004 & 0,000 & 0,9990 \\
11 & 1,003 & 0,002 & 0,9900 & 0,998 & 0,001 & 0,9988 \\
12 & 1,004 & 0,001 & 0,9973 & 1,008 & 0,001 & 0,9988 \\
\hline
\end{tabular}

Apenas o modelo 8 apresentou significância para o coeficiente $a_{2}$, enquanto que as combinações das variáveis $C$ e L para definição dos modelos permitiram ajustes iguais do coeficiente $a_{1}$ entre as equações 1 e 2; 4 e 5; 9 e 10 (Tabelas 6). Dessa forma, os modelos 2; 5 e 10 igualaram-se aos modelos 1, 4 e 9, respectivamente, e para evitar a duplicidade de resultados foram excluídos da etapa de validação.

A utilização de apenas uma variável de entrada em uma equação linear levou à um maior coeficiente angular ajustado, inclusive no modelo 8, que estima AFR a partir da largura da folha (Tabela 6). Para todos os modelos foram obtidos coeficientes de determinação $\left(R^{2}\right)$ superiores a 0,75 . Nesse caso, os menores valores foram observados nas equações $1 \mathrm{e}$ 4, sendo 0,7937; 0,7561 (AA) e 0,7840; 0,7608 (AJ), em contrapartida, as equações 8 e 11 apresentaram os maiores coeficientes de determinação: 0,9847 (AA) e 0,9710; 0,9712 (AJ) (Tabela 6).

Nenhum modelo superestimou a AFR, uma vez que todos os valores de MBE foram negativos, todavia as subestimativas foram inferiores a $26,4 \quad \mathrm{~cm}^{2}$. Independentemente do modelo, observou-se valores menores de MBE para estimativas de folhas de plantas que ainda não atingiram o estádio reprodutivo.
Tabela 6. Coeficientes ajustados $\left(a_{1}, a_{2}, b\right)$ e de determinação $\left(R^{2}\right)$ para os modelos de estimativa da área da folha de B. excelsa.

Table 6. Adjusted coefficients $\left(a_{1}, a_{2}, b\right)$ and determination coefficients $\left(\mathrm{R}^{2}\right)$ for the estimation models of the leaf blade area of B. excelsa.

\begin{tabular}{ccccc}
\hline \multicolumn{5}{c}{ Árvores Jovens - AJ } \\
\hline Equação & $\mathrm{a}_{1}$ & $\mathrm{a}_{2}$ & $\mathrm{~b}$ & $\mathrm{R}^{2}$ \\
\hline 1 & 7,8979 & - & - & 0,7937 \\
2 & 7,8979 & 0 & - & 0,7937 \\
3 & 0,5733 & - & 1,7724 & 0,9229 \\
4 & 22,6386 & - & - & 0,7561 \\
5 & 22,6386 & 0 & - & 0,7561 \\
6 & 2,7449 & - & 1,9074 & 0,9208 \\
7 & 0,7762 & - & - & 0,9847 \\
8 & 0,7705 & 1,8134 & - & 0,9847 \\
9 & 5,8806 & - & - & 0,8005 \\
10 & 5,8806 & 0 & - & 0,8005 \\
11 & 0,7997 & - & 0,9949 & 0,9847 \\
12 & 0,1970 & - & 1,9209 & 0,9673 \\
\hline \multicolumn{5}{c}{ Árvores Adultas - AA } \\
\hline Equação & $\mathrm{a}_{1}$ & $\mathrm{a}_{2}$ & $\mathrm{~b}$ & \\
\hline 1 & 8,5236 & - & - & 0,7840 \\
2 & 8,5236 & 0 & - & 0,7840 \\
3 & 0,6152 & - & 1,7790 & 0,9230 \\
4 & 22,0723 & - & - & 0,7608 \\
5 & 22,0723 & 0 & - & 0,7608 \\
6 & 2,2896 & - & 1,9418 & 0,9359 \\
7 & 0,8825 & - & - & 0,8909 \\
8 & 0,7543 & 5,1624 & - & 0,9710 \\
9 & 6,1761 & - & - & 0,7929 \\
10 & 6,1761 & 0 & - & 0,7929 \\
11 & 0,9334 & - & 0,9674 & 0,9712 \\
12 & 0,2353 & - & 1,8849 & 0,9614 \\
\hline
\end{tabular}

Tabela 7. Indicativos de desempenho estatísticos das equações de estimativa da área da folha com base em dimensões lineares.

Table 7. Statistical performance indicators of leaf area estimation equations based on linear dimensions.

\begin{tabular}{ccccc}
\hline Equação & MBA $\left(\mathrm{cm}^{2}\right)$ & RMSE $(\mathrm{cm})$ & $\mathrm{dW}$ & $\mathrm{VP}$ \\
\hline \multicolumn{5}{c}{ Árvores Jovens $-\mathrm{AJ}$} \\
\hline 1 & $-16,68$ & 53,76 & 0,9994 & 31 \\
3 & $-2,87$ & 33,40 & 0,9998 & 23 \\
4 & $-17,59$ & 58,96 & 0,9992 & 34 \\
6 & $-1,39$ & 30,23 & 0,9998 & 18 \\
7 & $-1,19$ & 18,19 & 0,9999 & 8 \\
8 & $-1,71$ & 18,17 & 0,9999 & 9 \\
9 & $-17,75$ & 53,57 & 0,9994 & 31 \\
11 & $-1,39$ & 18,19 & 0,9999 & 9 \\
12 & $-2,02$ & 24,32 & 0,9999 & 17 \\
\hline \multicolumn{5}{c}{ Árvores Adultas-AA } \\
1 & $-22,87$ & 48,18 & 0,9995 & 32 \\
3 & $-3,09$ & 24,55 & 0,9999 & 18 \\
4 & $-22,61$ & 50,51 & 0,9994 & 32 \\
6 & $-2,18$ & 31,03 & 0,9998 & 17 \\
7 & $-26,39$ & 33,49 & 0,9998 & 26 \\
8 & $-2,07$ & 14,36 & 0,9999 & 5 \\
9 & $-23,67$ & 47,36 & 0,9995 & 29 \\
11 & $-2,31$ & 14,41 & 0,9999 & 8 \\
12 & $-2,58$ & 15,61 & 0,9999 & 13 \\
\hline \multicolumn{5}{c}{}
\end{tabular}

Pelos valores de RMSE as equações 1, 4 e 9 tiveram maior espalhamento das estimativas em relação à AFR. Esse indicativo apresenta a amplitude média de valores estimados na variável dependente, mantendo o mesmo valor da variável independente. Nota-se uma inversão quando comparado ao MBE, visto que, as estimativas de AFR de folhas 
provenientes de plantas adultas apresentaram menores dispersões das estimativas (Tabela 7). $O$ índice de ajustamento de Willmott (dW) foi superior a 0,99 para todas as equações geradas, atestando assim, a exatidão e uma ótima concordância dos modelos entre AFE e AFR (Tabela 7).

A equação 7 apresentou o menor valor ponderado $(\mathrm{Vp})$ para obtenção de AFE de folhas provenientes de plantas jovens (8), enquanto que, para folhas provenientes de plantas adultas, as melhores estimativas de AFE foram dadas pela equação $8(\mathrm{Vp}=5)$ (Tabela 7). Dessa forma, subtraindo o erro (MBE), a área da folha de B. excelsa pode ser estimada com maior precisão e acurácia, utilizando medidas do comprimento e da largura no centro da folha, a partir das equações:

$$
\begin{aligned}
& \text { Para árvores Jovens } \\
& A F=(0,7762 * C * L)-1,19
\end{aligned}
$$

Para árvores adultas

$$
A F=[(0,7543 * C * L)+5,1624]-2,07 \text { (Equação 17) }
$$

em que: AF é a área foliar estimada $\left(\mathrm{cm}^{2}\right)$; C o comprimento da folha (cm); L a largura da folha $(\mathrm{cm})$.

Em alternativa a distinção entre idade da planta (matriz), foi realizada outra calibração dos modelos 8 e 11, que apresentam valores intermediários de $\mathrm{Vp}$ na primeira validação. Os coeficientes ajustados, o coeficiente de correlação, os indicativos estatísticos e o valor ponderado das equações 8 e 11 calibradas e validadas para todas as folhas são apresentados na Tabela 8.

Tabela 8. Coeficientes ajustados e indicativos dos modelos 8 e 11 para estimativa da área do limbo foliar de B. excelsa com base em medidas lineares.

Table 8. Adjusted and indicative coefficients of models 8 and 11 for estimation of leaf area of $B$. excelsa based on linear measurements.

\begin{tabular}{ccccccc}
\hline \multicolumn{7}{c}{ Modelo 8 } \\
\hline $\mathrm{a}_{1}$ & $\mathrm{a}_{2}$ & $\mathrm{R}^{2}$ & MBA & RMSE & $\mathrm{dW}$ & $\mathrm{VP}$ \\
0,7614 & 3,7620 & 0,9570 & $-1,95$ & 16,34 & 0,9999 & 6 \\
\hline \multicolumn{7}{c}{ Modelo 11 } \\
\hline $\mathrm{a}_{1}$ & $\mathrm{~b}$ & $\mathrm{R}^{2}$ & MBA & RMSE & $\mathrm{dW}$ & $\mathrm{VP}$ \\
0,8743 & 0,9790 & 0,9574 & $-1,84$ & 16,38 & 0,9999 & 5 \\
\hline
\end{tabular}

Nesse caso, subtraindo-se o erro (MBE), a área do limbo foliar de Bertholletia excelsa, independentemente da idade da planta (incluindo a fase de muda) pode ser estimada a partir da equação abaixo:

$$
A F=\left\{0,8743 *\left[(C * L)^{0,9790}\right]\right\}-1,84 \text { (Equação 18) }
$$

em que: AF é a área foliar estimada pela fórmula $\left(\mathrm{cm}^{2}\right)$; C o comprimento da folha $(\mathrm{cm})$; L a largura da folha $(\mathrm{cm})$.

\section{DISCUSSÃO}

Ficou evidente nas Tabelas 3 e 4 que os desvios e coeficientes de variação aumentaram para os conjuntos de folhas maiores (Classes II e III) e para árvores jovens. As dimensões lineares do limbo variam dentro dos limites genéticos de cada espécie, sendo determinadas pela própria idade foliar e também por fatores bióticos e abióticos do ambiente. Segundo Carvalho; Christoffoleti (2007) a disponibilidade de luz, estado fitossanitário e nutricional da planta podem alterar o tamanho das folhas. Esta heterogeneidade de classes poderia levar a ineficiência da modelagem, no entanto, esse erro foi minimizado diante da amplitude amostral (limites superiores e inferiores), da representatividade e homogeneidade entre os grupos de calibração e validação (demonstrados nas Figuras 3 e 4 e nas Tabelas 3 e 4).

Schmildt et al. (2016), conferiram credibilidade para ajustes de modelos de estimativa da área foliar para Coffea canephora com uma amostragem de 192 folhas, enquanto que, Koubouris et al. (2018), necessidade de 460 folhas de diferentes cultivares de Oliveira para obtenção de bons ajustes. Liu et al. (2017), estudaram modelos para estimativa de área foliar por método não destrutivo de 5 espécies florestais, e propuseram o número ótimo de amostras necessário para boa acurácia e erro razoável entre 40 a 60 folhas. Em todos esses casos, foram amostradas quantidades inferiores às deste estudo (750 folhas por idade da planta).

As características foliares são adequadas para o diagnóstico da planta, pois refletem às condições climáticas e edáficas, sendo importantes referênciais sobre o estado nutricional, a competição entre indivíduos e a micrometeorologia florestal (OKAJIMA et al., 2012). Dessa forma, considerando a média da classe III de ambas idades, plantas de castanheira-do-Brasil que não atingem dimensões lineares de 36,0 e 13,0 cm (comprimento e largura) e área foliar próxima de $370 \mathrm{~cm}^{2}$, podem estar sobre algum tipo de estresse, que inibe a total expansão foliar. Todavia, ressaltase que, na fase de viveiro (mudas), as folhas tendem a serem menores não devendo ser considerados esses valores como limitantes.

Os resultados expostos na Tabela 5 confirmaram que, aumentar o número de mensurações de largura em uma mesma folha in loco não aumenta a precisão da estimativa da área do limbo. Assim, as dimensões de comprimento e largura central são suficientes para boas estimativas de AFE. Os modelos com variáveis de entrada simples e de fácil coleta, como comprimento e largura, são mais indicados e desejáveis à campo, pois simplificam os procedimentos de medidas (MALDANER et al., 2009).

Lu et al., (2004) afirmaram que, quanto à modelagem da área foliar, na prática, o uso de equações de modelos lineares baseados em apenas uma dimensão linear é preferível por sua simplicidade para aplicação, principalmente no campo. No entanto, para Bertholletia excelsa utilizar uma única dimensão na modelagem diminui a precisão e exatidão da estimativa, haja visto os resultados inferiores obtidos nesse estudo com as equações de número 1 a 6 (Tabelas 2, 6 e 7).

O coeficiente linear não influenciou na estimativa da equação de primeiro grau exceto, quando são multiplicadas as variáveis de entrada $\left(\mathrm{C}^{*} \mathrm{~L}\right)$. De acordo com Liu et al. (2017), o produto entre comprimento e largura é o melhor parâmetro para predizer a área foliar.

Batista et al. (2014), encontraram coeficientes de determinação satisfatórios na estimativa da área foliar de $B$. excelsa, utilizando o modelo linear com base no produto do comprimento pela largura $[\mathrm{AFE}=0,7735(\mathrm{C} * \mathrm{~L})-7,4614] \mathrm{e} \mathrm{em}$ modelos geométricos com base no comprimento [AFE = $0,1814(\mathrm{C})^{2,052}$ e na largura $\left[\mathrm{AFE}=1,7674(\mathrm{~L})^{2,0784}\right.$. No entanto os autores só utilizaram como indicativo estatístico o coeficiente de determinação $\left(\mathrm{R}^{2}\right)$. 
Duas equações - uma linear e outra potencial, 8 e 11, apresentaram melhores ajustes para estimativa da área foliar, concordando com estudos de outros autores que apontaram a eficiência de modelos lineares $(y=a x+b)$ e potenciais $\left(y=a x^{b}\right)$ para descrever a relação entre as dimensões lineares e a área foliar. Tartaglia et al. (2016), determinou um modelo potencial para estimativa da área foliar de Brassica napus; Ribeiro et al. (2018), estudando Erythroxylum simonis e Keramatlou et al. (2015), estudando Juglans regia encontraram bons ajustes com modelos lineares a partir do produto entre comprimento e largura para determinação de área foliar. Salazar et al. (2018), também encontraram boa precisão e acurácia na estimativa de área foliar do cacau (Theobroma cacao) a partir de regressão linear.

Medri; Lleras (1979) demonstraram que da base para o ápice da copa da planta, as folhas tendem a diminuir de tamanho. Conforme observado nas Tabelas 3 e 4 e nas Figuras 3 e 4, podemos considerar que em média folhas de $B$. excelsa atingem seu limite máximo de área, ou seja, aquele programado geneticamente, quando próximas de $370 \mathrm{~cm}^{2}$. Sabendo que uma folha é autossustentável ao atingir $3 / 4$ de sua área total. Logo as folhas de Castanheira-do-Brasil deixam de ser drenos e passam a ser fontes de fotoassimilados ao ultrapassar $279,3 \mathrm{~cm}^{2}$.

No desenvolvimento inicial Bertholletia excelsa é comum que a planta esteja no sub-bosque da floresta ou em condições de viveiros, ambientes estes que propiciam condições de luz, vento e temperatura diferentes da fase adulta da planta, podendo levar a planta a desprender recursos em estruturas para interagir com o ambiente. $\mathrm{O}$ tecido que estava adaptado para incrementos na fotossíntese, pode ser gradualmente alterado com a presença de mecanismos de defesa contra estresse oxidativo, conforme muda a pressão no ambiente (COBLE et al., 2016). Essa mudança na estratégia de árvores jovens e adultas poderia causar diferenças estruturais e afetar os modelos empíricos calibrados para estimativa de área do limbo foliar. Contudo, a idade da planta de castanheira-do-Brasil não influenciou na modelagem de AFE (Tabela 8). As folhas da espécie mantêmse no formato oblongo, não apresentando polimorfismo foliar (Figura 5).

Brito-Rocha et al. (2016), analisaram Brosimum rubescens, Manilkara maxima, Pouteria caimito, Pouteria torta, Psidium cattleyanum, Symphonia globulifera e Tabebuia stenocalyx, e encontraram mudanças nos parâmetros estruturais recomendando que diferentes modelos empíricos devem ser desenvolvidos para estimar a área da folha durante os períodos ontogenéticos para as espécies supracitadas.

Wang et al. (2019), estudaram a influência do ciclo de vida na modelagem da folha de 8 espécies (Betula platyphylla, Betula costata, Tilia amurensis, Fraxinus mandshurica, Acer mono, Ulmus japonica, Ulmus laciniata e Acer tegmentosum) e recomendaram que o estádio de desenvolvimento deve ser considerado para construção de modelos, quando as folhas da espécie têm razão comprimento-largura menor que 1,5; em contraste quando maior que 1,5 , os autores não encontraram efeito significativo. Com base na análise desses autores, as folhas de B. excelsa apresentaram relações médias (C/LC) de 2,8 e 2,6 para AJ e AA (Tabela 4).

Mesmo permanecendo oblongas desde a emissão até a senescência, ocorrem diferenciações morfológicas no ápice da folha de B. excelsa ao longo do seu ciclo. Logo após a emissão, as folhas têm ápice apiculado, passando por acuminado, e quando maduras e completamente expandidas, têm ápice cuspidado. Esse padrão foi observado independentemente da idade da planta (Figura 5). Todavia, mesmo com essa alteração na terminação da folha, os modelos gerados foram satisfatórios para estimativa da área do limbo foliar de B. excelsa independente das idades da folha e da planta.

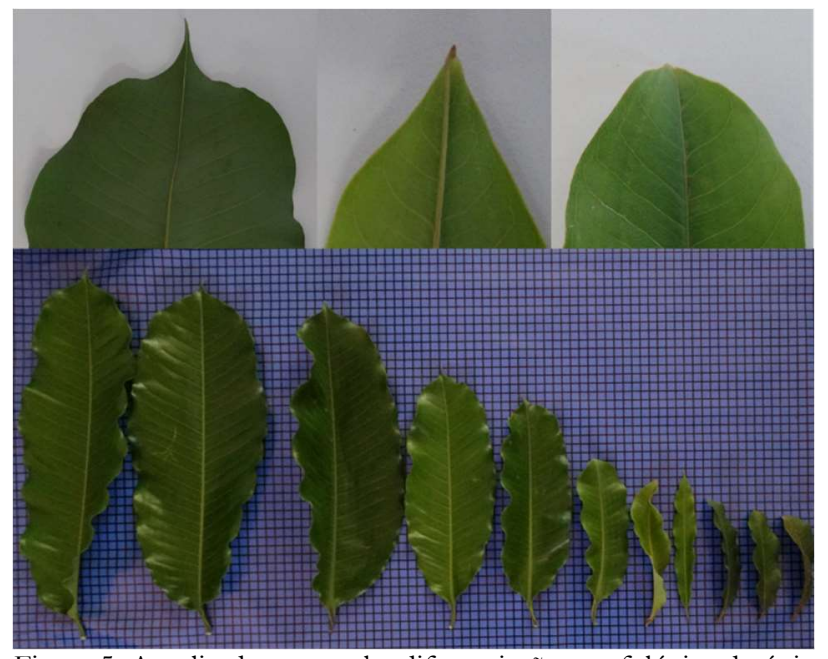

Figura 5. Amplitude amostral e diferenciação morfológica do ápice ao longo da maturação foliar de Bertholletia excelsa.

Figure 5. Sample amplitude and morphological differentiation of the apex along the leaf maturation of Bertholletia excelsa.

\section{CONCLUSÕES}

A estimativa da área da folha de Bertholletia excelsa deve ser realizada pela equação $\mathrm{AF}=\left\{0,8743 *[\mathrm{C} * \mathrm{~L}]^{0,9790}\right\}-1,84$, independentemente da idade da folha e da planta.

B. excelsa apresenta ápice foliar apiculado após a emissão, passando por acuminado e quando maduras ápice cuspidado.

\section{AGRADECIMENTOS}

O presente trabalho foi realizado com apoio da Coordenação de Aperfeiçoamento de Pessoal de Nível Superior - Brasil (CAPES) - Código de Financiamento 001.

\section{REFERÊNCIAS}

ABREU, M. C.; CECÍLIO, R. A.; PRUSKI, F. F.; SANTOS, G. R. dos; ALMEIDA, L. T. de; ZANETTI S. S. Critérios para Escolha de Distribuições de Probabilidades em Estudos de Eventos Extremos de Precipitação. Revista Brasileira de Meteorologia, São Paulo, v. 33, n. 4, p. 601-613, 2018. DOI: http://dx.doi.org/10.1590/01027786334004

BATISTA, K. D.; AMARO, G.; SOUZA, J. J. S.; PEDroso, C. Â.; AlbuQUerQue, T. C. S. Estimativa da área foliar de castanha do Brasil através de medidas lineares. Simpósio de crescimento de plantas. Resumos, Lavras: UFLA, 2014.

BENINCASA, M. M. P. Análise de crescimento de plantas: noções básicas. 2. ed. Jaboticabal: Funep, 2003. $41 \mathrm{p}$.

BRITO-ROCHA, E.; SCHILLING, A. C.; DOS ANJOS, L.; PIOTTO, D.; DALMOLIN A. C.; MIELKE M. S. Regression models for estimating leaf area of seedlings and adult individuals of Neotropical rainforest tree species. Brazilian Journal of Biology, São Carlos, v. 76, 
n. 4, p. 983-989, 2016. DOI: http://dx.doi.org/10.1590/1519-6984.05515

CABANEZ, P. A.; SILVA, S. F. da; PEREIRA, L. R.; MONTEIRO, E. de C. Estimativa da área foliar do biribazeiro através de modelos de regressão. Revista de Ciências Agroambientais, Alta Floresta, v. 12, n. 1, p. 51-55, 2014.

CARVALHO, S. J. P.; CHRISTOFFOLETI, P. J. Estimativa da Área Foliar de Cinco Espécies do Gênero Amaranthus usando Dimensões Lineares do Limbo Foliar. Planta Daninha, Viçosa-MG, v. 25, n. 2, p. 317-324, 2007.

COBLE, A. P.; WALL, B. V.; MAU A.; CAVALERI, M. A. How vertical patterns in leaf traits shift seasonally and the implications for modeling canopy photosynthesis in a temperate deciduous forest. Tree Physiology, Oxford, v. 36, n. 9, p. 1077-1091, 2016. DOI: https://dx.doi.org/10.1093/treephys/tpw043

CUSI-AUCA, E.; DIONISIO, L. F. S.; BARDALESLOZANO, R. M.; SCHWARTZ, G. Propagation of Brazil nut (Humb. y Bonpl) seedlings using seeds in mini-greenhouses. Revista Agro@mbiente On-line, Boa Vista, v. 12, n. 4, p. 300-313, 2018. DOI: http://dx.doi.org/10.18227/1982-8470ragro.v12i4.5222

DUCHELLE, A. E.; CRONKLETON, P.; KAINER, K.A.; GUANACOMA, G.; GEZAN, S. Resource theft in tropical forest communities: implications for non-timber management, livelihoods, and conservation. Ecology and Society, v. 16 , n. 1 , p. 35-55, 2011. DOI: https://dx.doi.org/10.5751/ES-03806-160104

FAVARIN, J. L.; DOURADO-NETO, D. Y.; GARCÍA, A. G.; VILLA NOVA, N. A.; FAVARIN, M. G. G. V. Equações para a estimativa do índice de área foliar do cafeeiro. Pesquisa Agropecuária Brasileira, Brasília, v. 37, n. 6, p. 769-773, 2002. DOI: http:/ /dx.doi.org/10.1590/S0100-204X2002000600005

FERREIRA, M. J.; GONCALVES, J. F. C.; FERRAZ, J. B. S. Crescimento e eficiência do uso da água de plantas jovens de Castanheira-da-Amazônia em área degradada e submetidas a adubação. Ciência Florestal, Santa Maria, v. 22, n. 2, p.393-401, abr./jun. 2012. DOI: http://dx.doi.org/10.5902/198050985747

FERREIRA, M. C.; ALANDIA, R. A.; CARVALHO, G. F. G; BAGGIO, M. V. Determinação de área foliar e retenção de líquido por folhas de café em pulverização a alto volume. Nucleus, Ituverava, v. 7, n. 1, p. 277-283, abr. 2010. DOI: http://dx.doi.org/10.3738/1982.2278316

JONES, D. A.; O'HARA, K. L.; BATLLES, J. J.; GERSONDE, R. F. Leaf area predction using three alternative sampling methods for seven Sierra Nevada conifer species. Forests, v. 6, n. 8, p. 2631-2654, 2015. DOI: https://dx.doi.org/10.3390/f6082631

KERAMATLOU, I.; SHARIFANI, M.; SABOURI, H.; ALIZADEH, M.; KAMKAR B. A simple linear model for leaf area estimation in Persian walnut (Juglans regia L.). Scientia Horticulturae, Amsterdam, v. 184, n. 1, p. 36-39, mar. 2015.2 DOI: https://dx.doi.org/10.1016/j.scienta.2014.12.017

KOUBOURIS, G.; BOURANIS, D.; VOGIATZIS, E.; NEJAD, A. R.; GIDAY, H.; TSANIKLIDIS, G.; LIGOXIGAKIS, E. K.; BLAZAKIS, K.; KALAITZIS, P.; FANOURAKIS, D. Leaf area estimation by considering leaf dimensions in olive tree. Scientia
Horticulturae, Amsterdam, v. 240, p. 440-445, out. 2018. DOI:

https://dx.doi.org/10.1016/j.scienta.2018.06.034

LEROY, C.; SAINT-ANDRÉ, L.; AUCLAIR, D. Practical methods for non-destructive measurement of tree leaf area. Agroforestry Systems, Dordrecht, v. 71, n. 3, p. 99 108, 2007. DOI: https://dx.doi.org/10.1007/s10457007-9077-2

LIMA, R. T.; SOUZA, P. J. O. P.; RODRIGUES, J. H.; LIMA, M. J. A. Modelos para estimativa da área foliar da mangueira utilizando medidas lineares. Revista Brasileira de Fruticultura, Jaboticabal, v. 34, n. 4, p. 974-980, dez. 2012. DOI: http:/ /dx.doi.org/10.1590/S0100-29452012000400003

LIU, Z.; ZHU, Y.; LI, F.; JIN, G. Non-destructively predicting leaf area, leaf mass and specific leaf area based on a linear mixed-effect model for broadleaf species. Ecological Indicators, v. 78, p. 340-350, 2017. DOI: https://doi.org/10.1016/j.ecolind.2017.03.025

LU, H. Y.; LU, C. T.; WEI, M.L.; CHAN, L. F. Comparison of Different Models for Nondestructive Leaf Area Estimation in Taro. Agronomy Journal, Madison, v. 96, n. 2, p. 448-453, 2004. DOI: https://dx.doi.org/10.2134/agronj2004.4480

MALDANER, I. C.; HELDWEIN, A. B.; LOOSE, L. H.; LUCAS, D. D. P.; GUSE, F. I.; BORTOLUZZI M. P. Modelos de determinação não-destrutiva da área foliar em girassol Ciência Rural, Santa Maria, v. 39, n. 5, p. 1356-1361, $2009 . \quad$ DOI: http:/ /dx.doi.org/10.1590/S0103-84782009000500008

MARTIM, A. R.; THOMAS, S. C. Size-dependent changes in leaf and wood chemical traits in two Caribbean rainforest trees. Tree Physiology, Oxford, v. 33, n. 12, p. 1338-1353, 2013.2 DOI: https://dx.doi.org/10.1093/treephys/tpt085

MAZZINI, R. B.; RIBEIRO, R. V.; PIO, R. M. A simple and non-destructive model for individual leaf area estimation in Citrus. Fruits, Cambridge, v. 65, n. 5, p. 269-275, out. 2010. DOI: https://dx.doi.org/10.1051/fruits/2010022

MEDRI, M. E.; LLERAS, E. Ecofisiologia de plantas da Amazônia. 2-Anatomia foliar e ecofisiologia de Bertholletia excelsa Humb. \& Benpl. (Castanha-do-pará) - Lecythidaceae. Acta Amazônica, Manaus, v. 9, n. 1 p. 15-23, 1979. DOI: http://dx.doi.org/10.1590/180943921979091015

MENG, F.; ZHANG, G.; LI, X.; NIKLAS, K. J.; SUN, S. Growth synchrony between leaves and stems during twig development differs among plant functional types of subtropical rainforest woody species. Tree Physiology, Oxford, v. 35, n. 6, p. 621-631, 2015. DOI: https://dx.doi.org/10.1093/treephys/tpv021

MONTEIRO, E. B.; SILVA, C. C.; SILVA, A. C.; SOUZA, A. P. Estimating emission of leaves seedlings forest in different shading levels, at conditions of Transition Amazon-Cerrado, Brazil. American Journal of Plant Sciences, v. 5, p. 2330-2341, 2014. DOI: https://dx.doi.org/10.4236/ajps.2014.515247

MONTEIRO, E. B.; SILVA, A. C.; SOUZA, A. P.; SILVA, C. C.; KAZAMA, V. S.; TANAKA, A. A. Statistical parameters to estimate the leaf area of native forest seedlings of genus Tabebuia and Handroanthus. Bioscience Journal, Uberlândia, v. 33, n. 4, p. 956-967, 2017. 
NEVES, E. de S.; WADT, L. H. de O.; GUEDES, M. C. Estrutura populacional e potencial para o manejo de Bertholletia excelsa (Bonpl.) em castanhais nativos do Acre e Amapá. Scientia Forestalis, Piracicaba, v. 44, n. 109, p. 19-31, 2016.2 DOI: http:/ /dx.doi.org/10.18671/scifor.v44n109.02

OKAJIMA, Y.; TANEDA, H.; NOGUCHI, K.; TERASHIMA I. Optimum Leaf Size Predicted by a Novel Leaf Energy Balance Model Incorporating Dependencies of Photosynthesis on Light and Temperature. Ecological Research, Tsukuba, v. 27, n. 2, p. 333-346, 2012. DOI: https://dx.doi.org/10.1007/s11284-011-0905-5

OLIVEIRA, F. A. Produção de feijão caupi em função da salinidade e regulador de crescimento. Revista Brasileira de Engenharia Agrícola e Ambiental, Campina Grande, v. 9, n. 11, p. 1049-1056, 2015. DOI: http://dx.doi.org/10.1590/18071929/agriambi.v19n11p1049-1056

PINTO, A. C. R. Modelos para estimativa de área foliar de Curcuma alismatifolia e Curcuma zedoaria. Bragantia, Campinas, v. 67, n. 2, p. 549-552, 2008. DOI: http://dx.doi.org/10.1590/S0006-87052008000200033

QUEIROZ, J. E.; SILVA, G. H.; SOUZA NETO, A. G. Avaliação estatística da área foliar através de modelos de equações em duas espécies florestais. Revista Verde de Agroecologia e Desenvolvimento Sustentável, Pombal, v. 8, n. 1, p. 146-153, 2013.

RIBEIRO, J. E. S.; BARBOSA, A. J. S.; ALBUQUERQUE, M. B. Leaf Area Estimate of Erythroxylum simonis Plowman by Linear Dimensions. Floresta e Ambiente, Seropédica, v. 25, n. 2, p. 243-250, 2018. DOI: http://dx.doi.org/10.1590/2179-8087.010817

SACHET, M. R.; PENSO, G. A.; PERTILlE, R. H.; GUERREZI, M. T.; CITADIN, I. Estimativa da área foliar de pessegueiro por método não-destrutivo. Ciência Rural, Santa Maria, v. 45, n. 12, p. 2161-2163, 2015. DOI: http://dx.doi.org/10.1590/0103$8478 \mathrm{cr} 20140185$

SALAZAR, J. C. S.; MELGAREJO, L. M.; BAUTISTA, E. H. D.; RIENZO, J. A. DI; CASANOVES, F. Nondestructive estimation of the leaf weight and leaf area in cacao (Theobroma cacao L.). Scientia Horticulturae, Amsterdam, v. 229, p. 19-24, 2018. DOI: https://dx.doi.org/10.1016/j.scienta.2017.10.034

SCHMILDT, E. R.; AMARAL, J. A. T.; SANTOS, J.S.; SCHMILDT, O. Allometric model for estimating leaf area in clonal varieties of coffee (Coffea canephora). Revista Ciência Agronômica, Fortaleza, v. 46, n. 4, p. 740-748, 2016. DOI: http://dx.doi.org/10.5935/18066690.20150061

SCOLES, R.; CANTO, M. S.; ALMEIDA, R. G.; VIEIRA, D. P. Sobrevivência e Frutificação de Bertholletia excelsa Bonpl. em Áreas Desmatadas em Oriximiná, Pará. Floresta e Ambiente, Seropédica, v. 23, n. 4, p. 555-564, 2016. DOI: http://dx.doi.org/10.1590/21798087.132015

SILVA, C. C. da; SOUZA, A. P. de; BOUVIÉ, L.; FERNEDA, B. G.; LEITE NETO, A.; MONTEIRO, E. B. Modelos alométricos para estimar a área do limbo foliar de teca. Nativa, Sinop, v. 8, n. 1, p. 129-136, 2020. DOI: http://dx.doi.org/10.31413/nativa.v8i1.7969
SOUZA, A. P.; MOTA, L. L.; ZAMADEI, T.; MARTIN, C. C.; ALMEIDA, F. T.; PAULINO, J. Classificação climática e balanço hídrico climatológico no estado de mato grosso. Nativa, Sinop, v. 1, n. 1, p. 34-43, 2013. DOI: http:/ /dx.doi.org/10.14583/23187670.v01n01a07

SOUZA, A. P.; SILVA, A. C.; LEONEL, S.; SOUZA, M. E.; TANAKA, A. A. Estimativas da área da folha de figueiras 'Roxo de Valinhos' usando dimensões lineares do limbo foliar. Ciência Rural, Santa Maria, v. 44, n. 7, p. 11721179, 2014. DOI: http://dx.doi.org/10.1590/0103$8478 \mathrm{cr} 20130699$

TARTAGLIA, F. DE L.; RIGHI, E. Z.; ROCHA, L. DA; LOOSE, L. H.; MALDANER, I. C.; HELDWEIN, A. B. Non-destructive models for leaf area determination in canola. Revista Brasileira de Engenharia Agrícola e Ambiental, Campina Grande, v. 20, n. 6, p. 551-556, 2016. DOI: http://dx.doi.org/10.1590/18071929/agriambi.v20n6p551-556

TONDJO, K.; BRANCHERIAU, L.; SABATIER, S.; KOKUTSE, A. D.; AKOSSOU, A.; KOKOU, K.; FOURCAUD, T. Non-destructive measurement of leaf area and dry biomass in Tectona grandis. Trees - Structure and Function, Berlin, v. 29, n. 5, p. 1625-1631, out. 2015. DOI: https://dx.doi.org/10.1007/s00468-015-1227-y

WANG, Y.; JIN, G.; SHI, B.; LIU, Z. Empirical models for measuring the leaf area and leaf mass across growing periods in broadleaf species with two life histories. Ecological Indicators, v. 102, p. 289-301, 2019. DOI: https://dx.doi.org/10.1016/j.ecolind.2019.02.041

WILLMOTT, C. J. On the validation of models. Physical Geography, v. 2, n. 2, p.184-194, 1981. DOI: https://doi.org/10.1080/02723646.1981.10642213 